\title{
Evaluation of Tourism Industry Efficiency in Pearl River Delta and its Influencing Factors in the Context of Happiness Industry
}

\author{
Jun Yuan, Wenshi Liang, Yanhong Liu* \\ Department of Leisure Tourism, Shenzhen University, Shenzhen, China \\ *Corresponding Author.
}

\begin{abstract}
In the context of happiness industry, this paper constructs the input-output index system of tourism industry efficiency, uses DEA model and Malmquist model to measure the tourism industry efficiency and its temporalspatial evolution of nine cities in Pearl River Delta from 2010 to 2016, and uses Grey relational model to analyze the influencing factors.The results show that, the overall tourism industry efficiency in Pearl River Delta reaches a relatively high level; the overall efficiency and technical efficiency showed a descending trend from inside out with the Pearl River Estuary economic circle as the core. The scale efficiency of tourism industry in Pearl River Delta showed a good trend, and the tourism elements input could satisfy the livelihood needs to a great extent. The total factor productivity of most cities in Pearl River Delta shows a growing trend, but the change trend differs in different areas. Impact of influencing factors of tourism industry efficiency differs in cities of different development degree and the impact of social benefit and environmental benefit indicators on tourism industry efficiency increases significantly. Based on the construction of happiness industry, cities in Pearl River Delta should take effective measures to improve the tourism industry efficiency.
\end{abstract}

Keywords:Tourism industry efficiency, DEA model, grey relational model, influencing factors, Pearl River Delta

\section{Introduction}

After entering the new era, fundamental changes have taken place in China's demand for tourism, namely to satisfy people's demand for a happy life. Tourism has already become an important index of rising living standard, symbolizing a happy life. In excellent geographical conditions and adjacent to Hong Kong and Macau, Pearl River Delta is one of the three urban agglomerations with the strongest comprehensive strength in China, and its tourism industry always takes the lead in development. Meanwhile, Pearl River Delta is also an important part in Guangdong-Hong Kong-Macao Greater Bay Area. It was clearly proposed in the Guidelines for the Development of Guangdong-Hong Kong-Macao Greater Bay Area to support cities in the Pearl River Delta to construct national tourism demonstration area, and jointly build an excellent social circle suitable for living, business and travel and offering good public services. All these indicate that tourism industry in the Pearl River Delta is now transforming from economy-oriented industry to happiness-oriented industry, and the ultimate development goal of the tourism industry is to improve the life quality and satisfy people's demands for good life. Under the new situation, how will the efficiency evaluation index system of traditional tourism industry be adjusted? What are the influencing factors of tourism industry in the context of happiness industry? The study on these problems will be of great significance for the tourism industrydevelopment in the new era.

As the research focus of economics and management science, efficiency problem also becomes one of the research focuses of the tourism discipline in recent years. Efficiency problem involves the inclusive growth of tourism industry, which is of great significance for the sustainable development of the tourism industry. Tourism industry efficiency is an effective index for measuring whether various social and tourism resources are properly utilized during the development of tourism at a specific economic and technological level. Foreign scholars launched the study on tourism efficiency in the middle of the 1990s, and the study was about the efficiency evaluation of tourism-related enterprises covering the hotelindustry[1-3],travel agency [4],tourism traffic, scenic spots[5,6], etc.

ISSN: 0010-8189

(c) CONVERTER 2021 
Research on tourism efficiency started late in China, at the beginning of the $21^{\text {st }}$ century.Chinese scholars pay more attention to the macro and meso level, and focus on different scales of regional tourism industry efficiency measurement and different types of tourism enterprise efficiency evaluation, etc. In some of the studies, researchers conducted empirical research on the tourism industry efficiency of Guangdong, Hubei, Jiangsu, Sichuan, etc. from the mesoscale based on DEA model and Malmquist index model, and analyzed the spatialtemporal differentiation and formation mechanism [7-11].

Although a series of progress has already been made in studies on tourism industry efficiency, there are still some deficiencies: firstly, evaluation of tourism industry efficiency emphasizes too much on economy-oriented tourism efficiency, and ignores social-oriented tourism efficiency. Theevaluation model and index system of tourism industry efficiency should make corresponding adjustment to the changes of the age. Secondly, there are few studies on the quantitative recognition and analysis of influencing factors. Existing studies just evaluate and analyze the tourism industry efficiency, but fail to recognize the influencing factors of tourism industry efficiency. This paper uses DEA model and Malmquist index model to measure and evaluate tourism industry efficiency and its spatial-temporal differentiation in various cities in Pearl River Delta in the context of happiness industry, and recognizes the influencing factors with Grey relational model, in order to supplement the theory system of evaluation of the efficiency of tourism and promote tourism industry efficiency in the Pearl River Delta.

\section{Methods and Data Sources}

\subsection{DEA model}

DEA is an effective method of evaluating multi-input and multi-output decision making units (DMU) [12]. In this paper, DEA is employed to evaluate the development efficiency of tourism industry in the Pearl River Delta. Suppose there are $n$ decision making units $(j=1,2,3, \ldots, n)$, each decision making unit $\mathrm{j}$ has $\mathrm{m}$ inputs and $\mathrm{s}$ outputs, $\left(X_{j}, Y_{j}\right)$ represents a combination of input and output vector. The DEA model (CRS model) based on constant returns to scale is shown below.

$$
\left\{\begin{array}{c}
\min \left[\theta-\varepsilon\left(\hat{e}^{T} S^{-}+e^{T} S^{+}\right)\right] \\
\text {s.t } \sum_{j=1}^{n} X_{j} \lambda_{j}+S^{-}=\theta X_{0} \\
\sum_{j=1}^{n} Y_{j} \lambda_{j}-S^{+}=Y_{0} \\
\lambda_{j} \geq 0, j=1, \ldots, n \\
S^{+} \geq 0, \quad S^{-} \geq 0
\end{array}\right.
$$

In Equation (1), $\theta(0<\theta \leq 1)$ is the factor input-output overall efficiency. The higher $\theta$ value is, the higher the overall efficiency of tourism will be. When $\theta=1$, it indicates that the overall tourism efficiency of the JTH region is at the optimum level. When $0.7 \leq \theta<1$, the overall efficiency is at a good level. When $0.5 \leq \theta<0.7$, the overall efficiency is at a medium level. When $\theta<0.5$, the overall efficiency is invalid. $S^{-}\left(\mathrm{S}^{-} \geq 0\right)$ is the slack variable, $\mathrm{S}^{+}$ $\left(S^{+} \geq 0\right)$ is the residual variable, $\varepsilon$ is a non-Archimedean infinitesimal quantity, $\lambda_{j}\left(\lambda_{j} \geq 0\right)$ is the weight variable. If the constraint conditions are added to the equation, Equation (1) can be transformed into the DEA model (VRS model) with variable returns to scale, which makes $\theta=\theta_{T E} \times \theta_{S E}$, where $\theta_{T E}$ is the pure technical efficiency of tourism and $\theta_{S E}$ is the scale efficiency of tourism. According to the research of relevant scholars [7,9], when $\theta_{T E}\left(\theta_{S E}\right)=1$, the efficiency is the highest. When $0.8 \leq \theta_{T E}\left(\theta_{S E}\right)<1$, the efficiency is good. When $0.6 \leq \theta_{T E}\left(\theta_{S E}\right)<0.8$, the efficiency is at a medium level; as $\theta_{T E}\left(\theta_{S E}\right)<0.6$, the efficiency is invalid.

2.2Malmquist model

ISSN: 0010-8189

(C) CONVERTER 2021 
The Malmquist index can be used for estimating the changes in tourism industry efficiency compared with the last year, and analyzing the dynamic change trend of tourism industry efficiency of different cities in Pearl River Delta [10]. This research introduces Malmquist index model to evaluate and compare the change trend of tourism industry efficiency of nine cities in Pearl River Delta.

$$
M I=\left[\frac{D^{t+1}\left(x^{t+1}, y^{t+1}\right)}{D^{t+1}\left(x^{t}, y^{t}\right)} \times \frac{D^{t}\left(x^{t+1}, y^{t+1}\right)}{D^{t}\left(x^{t}, y^{t}\right)}\right]^{\frac{1}{2}}
$$

$D^{t}\left(x^{t+1}, y^{t+1}\right)$ and $D^{t}\left(x^{t}, y^{t}\right)$ represent the distance between the DMU and the production frontier in period $t$ and period $\mathrm{t}+1$ when period $\mathrm{t}$ is taken as the technical reference, that is, the distance function, and the distance function of period $\mathrm{t}$ and period $t+1$ when period $t+1$ is taken as the technical reference, respectively. $M I>1$ represents an efficiency improvement. If $M I=1$, efficiency is unchanged. $M I<1$ means decreased efficiency [11].

\subsection{Grey relational model}

As a method of multi-factor statistical analysis, Grey relational model is usually used to analyze the influence degree of each factor on the results, so as to describe the relationship between independent variables and dependent variables [13]. The computing consists of five steps.

Step 1, decide reference sequence that reflects the system's behavior characteristics and comparison sequence that impacts the system behavior. This study takes the historical overall tourism efficiency of cities in Pearl River Delta as the reference sequence $x_{0}$, and factors impacting the tourism industry efficiency as the comparison sequence $x_{i}$ $(i=1,2,3 \ldots 7)$.

Step 2, dimensionless treatment is conducted for reference sequence and comparison sequence.

Step 3, calculate the Grey relational coefficient.

$$
\xi_{i}=\frac{\min _{i} \min _{k}^{\min }\left|x_{o}^{\prime}(k)-x_{i}^{\prime}(k)\right|+\rho \max _{i}^{\max } \max _{k}^{\max }\left|x_{o}^{\prime}(k)-x_{i}^{\prime}(k)\right|}{\left|x_{o}^{\prime}(k)-x_{i}^{\prime}(k)\right|+\rho \max _{i}^{\max }\left|x_{o}^{\prime}(k)-x_{i}^{\prime}(k)\right|}
$$

$\xi_{i}$ is the relational coefficient of $x_{o}^{\prime}$ and $x_{i}^{\prime}$ in time of $k, \rho$ is the resolution coefficient; between 0 and 1 , the smaller $\rho$ is, the greater the resolution will be. When $\rho=0.5$, and the relational degree is greater than 0.6 , it is considered that the two factors are in significant correlation.

Step 4, calculate the grey relational degree. There is more than one relational coefficient, so it needs to work out the mean by integrating the relational coefficients in different years. At this moment, formula of relational degree $r_{i}$ is as follows:

$$
r_{i}=\frac{1}{n} \sum_{i=1}^{n} \xi_{i}
$$

Step 5, rank the relational degree. The judgment of relational degree between independent variables and dependent variables focuses on the ranking of relational degree. If $r_{1}>r_{2}$, it means that the correlation between the reference sequence (dependent variable) $x_{0}$ and comparison sequence (dependent variable) $x_{1}$ is greater.

\subsection{Data sources}

All cities in Pearl River Delta are taken as the research area, including Guangzhou, Shenzhen, Zhuhai, Foshan, Huizhou, Dongguan, Zhongshan, Jiangmen and Zhaoqing. When selecting evaluation index of tourism industry efficiency, attention shall be paid to the following three points: firstly, select representative tourism input and 
output indexes that are closely related to the "happiness industry"; secondly, two times of total indexes shall not exceed total DMU units, according to the principle of DEA model; thirdly, data shall be available and accurate. Indexes shall be selected according to the above principles. Since there are 9 cities in Pearl River Delta, the total number of input and output indexes is 4 at most. According to the above principles, tourism resource attraction and fixed asset investment of tourism enterprises are selected as the input indexes, and the total tourism income and happy life index are selected as output indexes.

When selecting influencing factors of tourism industry efficiency, indexes shall be closelyrelated to the happiness industry. Considering the economic benefit, social benefit and environmental benefit of tourism, economic development level $\left(x_{1}\right)$, status of tourism industry $\left(x_{2}\right)$, tourism investment level $\left(x_{3}\right)$, tourism human resource $\left(x_{4}\right)$, tourism resources quality $\left(x_{5}\right)$, tourism traffic condition $\left(x_{6}\right)$, tourism environment quality $\left(x_{7}\right)$ are eventually selected as the influencing factors of tourism industry efficiency.

The above data are from Guangdong Statistical Yearbook (2011-2017), Guangdong Tourism Yearbook (20112017), statistical yearbook of nine cities in Pearl River Delta, official websites of Guangdong Department of Culture and Tourism and Tourism Bureau of all cities, Statistical Bulletin on national economy and social development, Report on the Overall Performance of Local Governments in Guangdong, etc. All data take municipal district as the statistical range.

\section{Results and Discussion}

\subsection{Characteristics and temporal-spatial evolution of tourism industry efficiency}

The average overall tourism industry efficiency in Pearl River Delta from 2010 to 2016 was 0.802, which was good, and it was close to 1 in most years in Shenzhen, Zhuhai, Dongguan and Zhongshan. The four cities are all in the core economic circle of Pearl River Estuary. In recent years, the public sense of happiness grows and tourism industry efficiency increases, due to the reinforcement of the integration of tourism resources and industry amalgamation, optimization of tourism environment, and improvement of public service functions for tourism in recent years; The average overall tourism industry efficiency in Guangzhou and Zhaoqing was 0.826 and 0.925 respectively, at a good level, while that of Foshan, Huizhou and Jiangmen was $0.538,0.425$ and 0.562 respectively, at a medium and invalid level. The three cities launched tourism industry early, as the region of mature tourism industry. But currently, the development enters the lag phase, with redundancy and resource allocation problems, and the overall tourism industry efficiency is low. From the perspective of temporal-spatial evolution, the overall efficiency of most cities in Pearl River Delta keeps growing, but it is relatively low or invalid in cities outside the Pearl River Delta. The overall efficiency declines gradually from inside out with the Pearl River Estuary economic circle, Guangdong-Hong Kong-Macao Greater Bay Area as the core(Fig.1, Table 1).
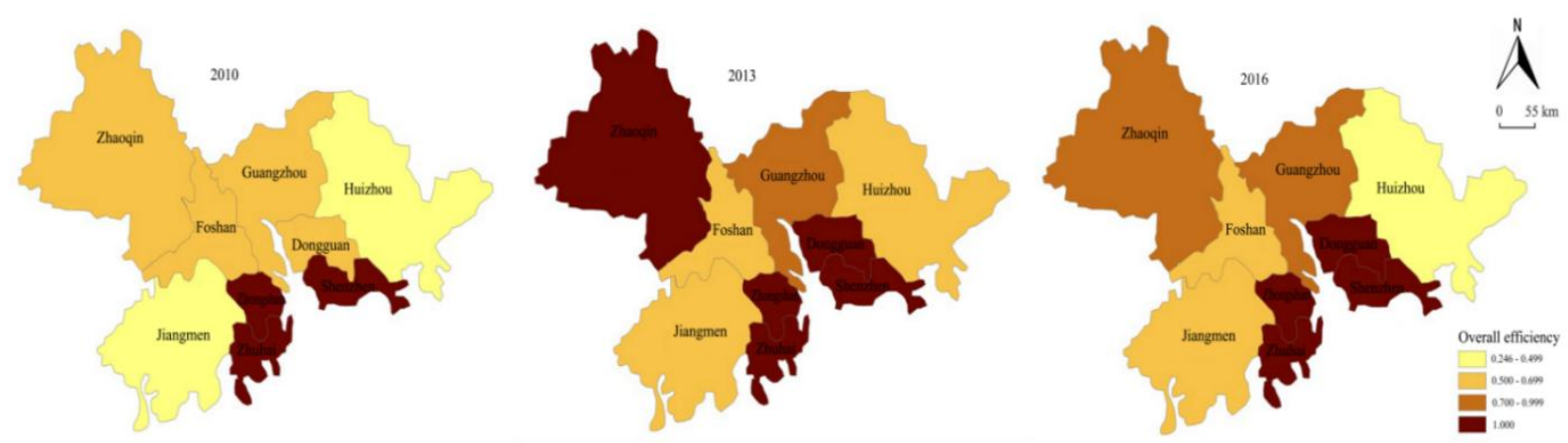

Fig. 1: Spatial-temporal evolution of overall tourism industry efficiency during 2010-2016

ISSN: 0010-8189 
Table 1Overall tourism industry efficiency of different cities in Pearl River Delta during 2010-2016

\begin{tabular}{ccccccccc}
\hline & $\mathbf{2 0 1 0}$ & $\mathbf{2 0 1 1}$ & $\mathbf{2 0 1 2}$ & $\mathbf{2 0 1 3}$ & $\mathbf{2 0 1 4}$ & $\mathbf{2 0 1 5}$ & $\mathbf{2 0 1 6}$ & Average \\
\hline Guangzhou & 0.599 & 1 & 1 & 0.767 & 0.719 & 0.753 & 0.947 & 0.826 \\
Shenzhen & 1 & 0.992 & 1 & 1 & 1 & 1 & 1 & 0.999 \\
Zhuhai & 1 & 1 & 1 & 1 & 1 & 1 & 1 & 1 \\
Foshan & 0.501 & 0.568 & 0.470 & 0.558 & 0.598 & 0.461 & 0.610 & 0.538 \\
Huizhou & 0.246 & 0.330 & 0.402 & 0.625 & 0.616 & 0.384 & 0.369 & 0.425 \\
Dongguan & 0.653 & 1 & 1 & 1 & 1 & 1 & 1 & 0.950 \\
Zhongshan & 1 & 1 & 1 & 1 & 1 & 0.997 & 1 & 1 \\
Jiangmen & 0.402 & 0.658 & 0.547 & 0.600 & 0.668 & 0.555 & 0.503 & 0.562 \\
Zhaoqin & 0.593 & 0.879 & 1 & 1 & 1 & 1 & 1 & 0.925 \\
\hline
\end{tabular}

The averagetechnical efficiency in Pearl River Delta from 2010 to 2016 was 0.851 , which was good. Specifically, the technical efficiency in Guangzhou, Shenzhen, Zhuhai and Zhongshan was always at an optimal level, and it was above 0.9 in Dongguan and Zhaoqing, suggesting that the six cities have a strong ability to utilize existing technology and allocates resources properly. It was 0.689 in Foshan, at medium level; but it was lower than 0.6 in Huizhou and Jiangmen, which was invalid, reflecting that the three cities were incapable of technology utilization, and shall enlarge the input in tourism technology. From the perspective of temporal-spatial evolution of technical efficiency, Guangzhou, Shenzhen, Zhuhai and Zhongshan were optimal in 2010, Dongguan and Zhaoqing were at medium level, but Foshan, Huizhou and Jiangmen were invalid; in 2013, Guangzhou, Shenzhen, Zhuhai, Foshan, Dongguan, Zhongshan and Zhaoqing were at an optimal level, Foshan was at a medium level, but Huizhou and Jiangmen were invalid; in 2016, Guangzhou, Shenzhen, Zhuhai, Dongguan, Zhongshan and Zhaoqing were at an optimal level, Foshan was at a medium level, but Huizhou and Jiangmen were invalid. In conclusion, the overall technical efficiency of tourism industry in Pearl River Delta was good since 2010, and tourism technical efficiency grew constantly. Most cities were optimal or grew better in tourism resource allocation and technology utilization, and only a few cities were invalid. The technical efficiency cities close to the center of Pearl River Delta had higher technical efficiency than outer cities, being more advantageous in the acquisition of resources (Fig.2).
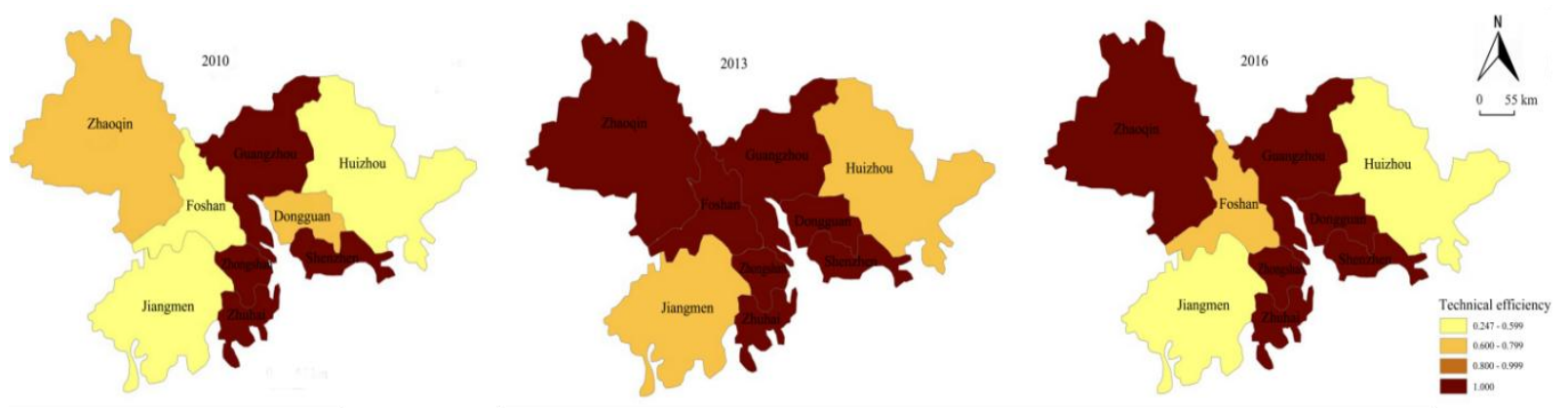

Fig. 2: Spatial-temporal evolution of technical tourism industry efficiency during 2010-2016

Tourism scale efficiency represents the effectiveness of production scale, and returns to scale is impacted by the increase ratio of tourism input and output. From 2010 to 2016, the average tourism scale efficiency in Pearl River Delta was 0.954, which was at a high level. Seen from the temporal-spatial evolution of scale efficiency(Fig.3), in 2010, all cities excluding Guangzhou reached an optimal level in tourism scale efficiency. In 2016, all cities reached an optimal level. On the whole, the tourism scale efficiency in Pearl River Delta presented a good trend, and tourism factor input can satisfy people's livelihood needs to a great extent, and the public sense of happiness and satisfaction ranked top in Guangdong. Seen from the temporal-spatial evolution of returns to scale(Table 2), it remained basically unchanged in Shenzhen, Zhuhai, Zhongshan, Dongguan and Zhaoqing from 2010 to 2016, and

ISSN: 0010-8189

(C) CONVERTER 2021 
tourism factor input could just maximize the livelihood income of tourism, in the best scale stage. The returns to scale in Guangzhou always decreased, meaning that the input of tourism factors had exceeded the digestion ability of the city, and resource and factor input was redundant. The returns to scale in Foshan, Huizhou and Jiangmen increased at the beginning but decreased in the middle to late stage, suggesting that tourism factor input was redundant, and these cities shall narrow the scale of tourism input, and focus on improving the development quality of tourism industry.
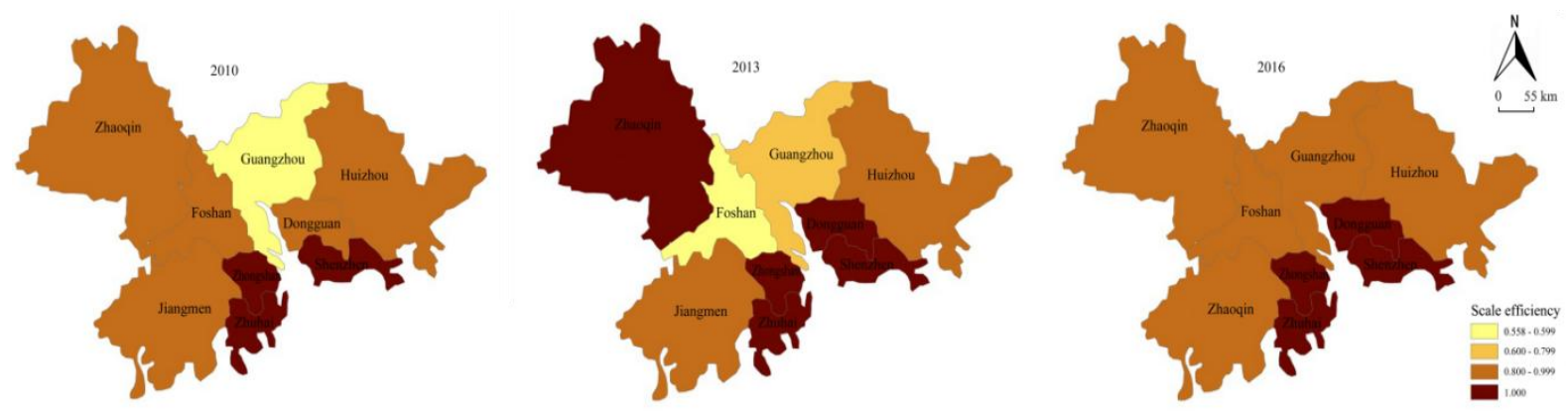

Fig. 3: Spatial-temporal evolution of scale tourism industry efficiency during 2010-2016

Table 2 Decomposition efficiency of tourism industry of various cities in Pearl River Delta during 2010-2016

\begin{tabular}{|c|c|c|c|c|c|c|c|c|c|}
\hline & \multicolumn{3}{|c|}{2010} & \multicolumn{3}{|c|}{2013} & \multicolumn{3}{|c|}{2016} \\
\hline & $\begin{array}{l}\text { Technical } \\
\text { efficiency }\end{array}$ & $\begin{array}{c}\text { Scale } \\
\text { efficiency }\end{array}$ & $\begin{array}{l}\text { Returns } \\
\text { to Scale }\end{array}$ & $\begin{array}{l}\text { Technical } \\
\text { efficiency }\end{array}$ & $\begin{array}{c}\text { Scale } \\
\text { efficiency }\end{array}$ & $\begin{array}{l}\text { Returns } \\
\text { to Scale }\end{array}$ & $\begin{array}{l}\text { Technical } \\
\text { efficiency }\end{array}$ & $\begin{array}{c}\text { Scale } \\
\text { efficiency }\end{array}$ & $\begin{array}{l}\text { Returns to } \\
\text { Scale }\end{array}$ \\
\hline Guangzhou & 1 & 0.599 & DRS & 1 & 0.767 & DRS & 1 & 0.947 & DRS \\
\hline Shenzhen & 1 & 1 & - & 1 & 1 & - & 1 & 1 & - \\
\hline Zhuhai & 1 & 1 & - & 1 & 1 & - & 1 & 1 & - \\
\hline Foshan & 0.508 & 0.987 & IRS & 1 & 0.558 & DRS & 0.612 & 0.997 & DRS \\
\hline Huizhou & 0.247 & 0.997 & IRS & 0.632 & 0.989 & DRS & 0.374 & 0.986 & DRS \\
\hline Dongguan & 0.659 & 0.990 & IRS & 1 & 1 & - & 1 & 1 & - \\
\hline Zhongshan & 1 & 1 & - & 1 & 1 & - & 1 & 1 & - \\
\hline Jiangmen & 0.423 & 0.950 & IRS & 0.617 & 0.973 & DRS & 0.516 & 0.974 & DRS \\
\hline Zhaoqin & 0.631 & 0.940 & IRS & 1 & 1 & - & 1 & 1 & - \\
\hline
\end{tabular}

3.2Analysis of change degree of tourism industry efficiency

Nine cities in Pearl River Delta showed different change trends in tourism industry efficiency from 2010 to 2016 (Table 3). The tourist efficiency of most cities kept improving, and the development of tourism industry can allocate resources properly, improve life quality, and meet people's demand for happy life. Specifically, MI in Guangzhou, Shenzhen, Zhuhai, Dongguan, and Zhongshan had been above 1 for 4 years, and the tourism industry efficiency kept growing. Only in Huizhou, MI was smaller than 1 for four years, and the overall tourism industry efficiency presented a declining trend, since Huizhou developed tourism resources early, with redundant tourism factor input, and the development of tourism industry confronts continuous technological innovation and progress. As a result, the total factor productivity presented a declining trend. MI of Foshan, Jiangmen and Zhaoqing fluctuated over the past years, but the overall tourism efficiency presented a growing trend. In general, most cities in Pearl River Delta attached great importance to the continuous innovation and progress of tourism technology, with strong tourism industry development competitiveness, under the impact of "the Belt and Road" and construction of Guangdong-Hong Kong-Macao Greater Bay Area, and driven by diversified market demand of tourism and government's livelihood-oriented tourism supply. The total factor productivity has presented a growing trend since 2010 . 
Table 3 Change degree of tourism industry efficiency of various cities in Pearl River Delta

\begin{tabular}{ccccccc}
\hline & $\mathbf{2 0 1 0 - 2 0 1 1}$ & $\mathbf{2 0 1 1 - 2 0 1 2}$ & $\mathbf{2 0 1 2 - 2 0 1 3}$ & $\mathbf{2 0 1 3 - 2 0 1 4}$ & $\mathbf{2 0 1 4 - 2 0 1 5}$ & $\mathbf{2 0 1 5 - 2 0 1 6}$ \\
\hline Guangzhou & 1.169 & 1.132 & 0.825 & 1.069 & 1.046 & 1.327 \\
Shenzhen & 0.762 & 1.097 & 1.043 & 1.184 & 1.114 & 1.048 \\
Zhuhai & 0.478 & 0.831 & 1.029 & 0.854 & 1.010 & 1.148 \\
Foshan & 0.858 & 0.750 & 1.121 & 1.106 & 0.971 & 1.385 \\
Huizhou & 1.014 & 0.962 & 1.223 & 0.939 & 0.843 & 0.980 \\
Dongguan & 1.084 & 0.791 & 0.869 & 1.356 & 1.288 & 1.299 \\
Zhongshan & 1.011 & 0.740 & 1.016 & 0.812 & 1.211 & 1.292 \\
Jiangmen & 0.630 & 0.654 & 0.981 & 1.016 & 1.016 & 1.014 \\
Zhaoqin & 1.099 & 0.970 & 0.786 & 0.890 & 1.279 & 1.036 \\
\hline
\end{tabular}

3.3Analysis of influencing factors of tourism industry efficiency

Grey relational degree of all measurement indexes in Pearl River Delta was close or greater than 0.6, suggesting that selected influencing factors had significant impacts on the tourism efficiency in Pearl River Delta. In which, among the seven influencing factors of tourism industry efficiency in Pearl River Delta, grey relational degree ranks in a descending order as follows: tourism environment quality $\left(x_{7}\right)$, status of tourism industry $\left(x_{2}\right)$, tourism traffic condition $\left(x_{6}\right)$, tourism resource quality $\left(x_{5}\right)$, tourism human resource $\left(x_{4}\right)$, economic development level $\left(x_{1}\right)$, tourism investment level $\left(x_{3}\right)$. There are certain differences in the impact of above factors when it comes to different cities in Pearl River Delta (Table 4, Table 5).

(1) Tourism environment quality $\left(x_{7}\right)$. The relational degree between tourism environment quality and tourism industry efficiency in Shenzhen, Zhuhai and Zhaoqing is evidently higher than other cities in Pearl River Delta. As core cities in Pearl River Delta, Shenzhen and Zhuhai have beautiful city appearance, and good ecological environment is the core attraction of tourism. Zhaoqing is situated near the mountain and by the water, and the advantaged natural and cultural environment contribute to the charm and core competitiveness of tourism. Urban tourism environment quality significantly impacts tourists' satisfaction, local residents' sense of happiness and tourism industry efficiency under the view of livelihood.

(2) Status of tourism industry $\left(x_{2}\right)$. The relational degree between the status of tourism industry and tourism industry efficiency in Foshan, Dongguan, Zhaoqing and Guangzhou is high, and the proportion of tourism industry in GDP increases year by year. Especially, these cities pay high attention to the development of tourism industry from the perspective of improving people's well-being, and develop the tourism industry to a strategic pillar industry of national economy and happiness industry that makes people more satisfied. The status and positioning of tourism industry have a great impact on the tourism industry efficiency.

(3) Tourism traffic condition $\left(x_{6}\right)$. The relational degree between tourism traffic condition and tourism industry efficiency in Zhuhai, Foshan, Dongguan, Zhaoqing, and Zhongshan is relatively high. Tourism development under the perspective of "happiness industry" sets higher requirements on public facilities like the traffic system. Extensive traffic network can not only enhance the accessibility of urban tourism, but also increase the convenience of traffic links and promote the integrated development of urban tourism in Guangdong-Hong KongMacao Greater Bay Area. Besides, the indirect economic and social benefits are immeasurable, which also impact the tourism industry efficiency.

(4) Tourism resource quality $\left(x_{5}\right)$. The relational degree between tourism resource quality and tourism industry efficiency in Zhaoqing, Shenzhen, Dongguan and Huizhou is relatively high. Zhaoqing and Huizhou have long been tourism cities in Pearl River Delta, with abundant tourism resources, and tourism attraction also becomes the

ISSN: 0010-8189 
key to tourism economic income. Shenzhen and Dongguan enjoy developed urban tourism and leisure tourism. With the proposal of all-for-one tourism, tourism development emphasizes more on quality and sharing with and benefiting the people. The tourism resource quality in these cities has certain impact on the tourism industry efficiency from the perspective of economic and social benefits.

(5) Tourism human resource $\left(x_{4}\right)$ and economic development level $\left(x_{1}\right)$. Impact of the two factors on the tourism industry efficiency in Pearl River Delta is of the same level, and the two factors have certain relation with the tourism industry efficiency. In the development of tourism industry in Pearl River Delta, the relational degree between tourism human resource input and tourism industry efficiency in Huizhou, Guangzhou, Dongguan, and Foshan is relatively high, while the relational degree between the economic development level and tourism industry efficiency in less developed cities like Huizhou and Zhaoqing is also relatively high, and economic factors have greater impacts on tourism industry efficiency. So economic benefit is still the focus of attention during the development of tourism industry in these cities.

(6) Tourism investment level $\left(x_{3}\right)$. The relational degree between tourism investment level and tourism industry efficiency in Pearl River Delta is the smallest, and the impact on other cities in Pearl River Delta excluding Zhongshan and Jiangmen is not evident. Tourism investment is an important premise of the development of tourism economy, playing a certain role in an early stage. But with the transition to happiness-oriented industry, economic benefit is no longer the only standard of measuring the tourism efficiency. Instead, attention is also paid to the social benefit and economic benefit. Furthermore, in the late stage of urban tourism development, if resources are not allocated properly, too many tourism inputs would also result in the redundancy of resource factors.

Table 4 Relational degree of influencing factors of tourism industry efficiency during 2010-2016

\begin{tabular}{cccccccc}
\hline & $\boldsymbol{r}_{\mathbf{1}}$ & $\boldsymbol{r}_{\mathbf{2}}$ & $\boldsymbol{r}_{\mathbf{3}}$ & $\boldsymbol{r}_{\mathbf{4}}$ & $\boldsymbol{r}_{\mathbf{5}}$ & $\boldsymbol{r}_{\mathbf{6}}$ & $\boldsymbol{r}_{\mathbf{7}}$ \\
\hline Pearl River Delta & 0.602 & 0.658 & 0.594 & 0.608 & 0.612 & 0.642 & 0.699 \\
Guangzhou & 0.622 & 0.654 & 0.591 & 0.659 & 0.617 & 0.595 & 0.516 \\
Shenzhen & 0.642 & 0.634 & 0.471 & 0.648 & 0.652 & 0.600 & 0.784 \\
Zhuhai & 0.582 & 0.496 & 0.593 & 0.593 & 0.457 & 0.672 & 0.646 \\
Foshan & 0.644 & 0.699 & 0.564 & 0.652 & 0.639 & 0.660 & 0.595 \\
Huizhou & 0.726 & 0.536 & 0.563 & 0.707 & 0.629 & 0.592 & 0.604 \\
Dongguan & 0.640 & 0.805 & 0.421 & 0.697 & 0.696 & 0.676 & 0.578 \\
Zhongshan & 0.537 & 0.568 & 0.779 & 0.481 & 0.469 & 0.619 & 0.507 \\
Jiangmen & 0.641 & 0.600 & 0.689 & 0.574 & 0.604 & 0.605 & 0.573 \\
Zhaoqin & 0.701 & 0.681 & 0.485 & 0.592 & 0.824 & 0.704 & 0.901 \\
\hline
\end{tabular}

Table 5 Relational degree rank of influencing factors of tourism industry efficiency during 2010-2016

\begin{tabular}{c|c|c|c|c|c|c|c}
\hline & $\boldsymbol{r}_{\mathbf{1}}$ & $\boldsymbol{r}_{\mathbf{2}}$ & $\boldsymbol{r}_{\mathbf{3}}$ & $\boldsymbol{r}_{\mathbf{4}}$ & $\boldsymbol{r}_{\mathbf{5}}$ & $\boldsymbol{r}_{\mathbf{6}}$ & $\boldsymbol{r}_{\mathbf{7}}$ \\
\hline Pearl River Delta & 6 & 2 & 7 & 5 & 4 & 3 & 1 \\
\hline Guangzhou & 3 & 2 & 6 & 1 & 4 & 5 & 7 \\
\hline Shenzhen & 4 & 5 & 7 & 3 & 2 & 6 & 1 \\
\hline Zhuhai & 5 & 6 & 4 & 3 & 7 & 1 & 2 \\
\hline Foshan & 4 & 1 & 7 & 3 & 5 & 2 & 6 \\
\hline Huizhou & 1 & 7 & 6 & 2 & 3 & 5 & 4 \\
\hline Dongguan & 5 & 1 & 7 & 2 & 3 & 5 & 6 \\
\hline Zhongshan & 4 & 3 & 1 & 6 & 7 & 2 & 5 \\
\hline Jiangmen & 2 & 5 & 1 & 6 & 4 & 3 & 7 \\
\hline Zhaoqin & 4 & 5 & 7 & 6 & 2 & 3 & 1 \\
\hline & & & & & &
\end{tabular}

ISSN: 0010-8189

(C) CONVERTER 2021 


\section{Conclusions}

This study constructs a tourism industry efficiency evaluation index system from the perspective of happiness and livelihood. It also analyzes the temporal-spatial evolution of tourism industry efficiency in nine cities in Pearl River Delta from 2010 to 2016 using DEA model, Malmquist index model and grey relational model, discusses its influencing factors and puts forward improvement policies. Empirical results have shown that the overall efficiency, technical efficiency and scale efficiency of tourism industry in Pearl River Delta reached a relatively high level from 2010 to 2016, and tourism industry efficiency also presented a growing trend, but there are certain differences among cities: the overall efficiency and technical efficiency of tourism industry in central cities were remarkably higher than that of outer cities in Pearl River Delta, presenting a descending trend from inside out with the Pearl River Estuary economic circle, Guangdong-Hong Kong-Macao Greater Bay Area as the core. The scale efficiency of tourism industry in Pearl River Delta presented a good trend, and reached an optimal or close-tooptimal state in 2016. Tourism factor input could satisfy the livelihood needs to a great extent. Total factor productivity of most cities in Pearl River Delta presented a growing trend, but the change trend differs in cities. Influencing factors of tourism industry efficiency in Pearl River Delta ranks in a descending order as follows: tourism environment quality, status of tourism industry, tourism traffic condition, tourism resource quality, tourism human resource, economic development level, tourism investment level. Under the construction of happiness industry, impact of social benefits and environmental benefits indexes on tourism industry efficiency increases significantly.

Research results are of direct guiding significance for the formulation of policies for the development of tourism industry by government sectors and improvement of tourism industry efficiency. Above all, in the context of happiness industry, attention is paid to the overall benefits in the development of tourism industry in Pearl River Delta, and the evaluation of tourism industry efficiency can help accurately estimate the development level and change trend of tourism industry efficiency in all cities, and provide reference for the improvement of tourism industry efficiency. Meanwhile, the impact of indexes reflecting the environmental benefit and social benefit on the tourism industry efficiency is significantly reinforced, suggesting that in the context of the construction of "happiness industry", more attention shall be paid to the tourism development quality in the development of tourism industry in Pearl River Delta, focusing on the public benefits: make planning for the tourism industry by centering on people's happiness, create an excellent tourism environment, improve tourism service system, and boost the transformation and escalation of tourism industry. In addition, cities are of different development degrees and stages, impact of influencing factors also differ, so different optimization and adjustment strategies shall be formulated to improve the tourism industry efficiency in Pearl River Delta. Powerful cities like Guangzhou, Shenzhen, Zhuhai, Dongguan, Zhongshan, Zhaoqing, etc. shall pay more attention to the social benefits and environmental benefits of tourism development, focus on the economic growth quality and lead the high-quality development of tourism industry in the Guangdong-Hong Kong-Macao Greater Bay Area; growing cities like Foshan and Jiangmen shall focus on optimizing the tourism factor input, integrate leisure tourism resources, improve resource allocation and technology utilization level of tourism industry. Growth stagnant city like Huizhou shall focus on improving the technological innovation capacity and people's demand for leisure tourism, to deepen the tourism supply side reform, and realize the leapfrog development of tourism.

\section{Acknowledgements}

This research was supported by the Philosophy and Social Sciences of Guangdong Province "13th Five-Year" Project Planning Foundation (GD16CGL14).

\section{References}

[1] C.M. Richard, A.D. David,"Evaluating a hotel GM's performance: A case in Benchmarking," Cornell Hotel Restaurant and Administration Quarterly,vol. 36, no. 5, pp. 30-3, 1995.

ISSN: 0010-8189 
[2] I.A.Randy, F. Mary, X. Yi, M. Frank, "Measuring efficiency in the hotel industry: a stochastic frontier approach,’International Journal of Hospitality Management, vol. 18, no.1, pp. 40-57, 1999.

[3] A. Ali,S. Hsin-Vonn, "The efficiency of the hotel industry in Singapore,"Tourism Management, vol. 37, pp. 31-34, 2013.

[4] F.Ramón,"Efficiency of travel agencies: a case study of Alicante, Spain," Tourism Management, vol. 32, no. 1, pp.75-87, 2011.

[5] B. Valentina, L. Gianni, "Data envelopment analysis approach to the assessment of natural park's economic efficiency and sustainability: the case of Italian natural parks," Sustainable Development,vol. 14, no. 4, pp. 277-286, 2006.

[6] A.S. Aurkene, Z. Mikel, R. Fidel, et al., "Obtaining the efficiency of tourism destination website based on data envelopment analysis," Procedia-Social and Behavioral Sciences, vol. 175, no. 12, pp.58-65, 2015.

[7] Y.l. Fang, Z.F. Huang,F.Wang, "Spatio-temporal Evolution of Provincial Tourism Efficiency and its Club Convergence in the Chinese Mainland," Progress in Geography, vol. 37, no. 10, pp.1392-1404, 2018.

[8] M.Z. Liang, T.T. Yi,L. Bin, "Study on the evolution model of tourism efficiency based on DEA-MI," Tourism Tribune, vol. 28, pp.5, pp. 53-61, 2013.

[9] H.Q. Li, L.Wang, "Evaluation of tourism efficiency in Hubei province based on DEA model," Statistics \& Decision, vol. 2, pp. 65-67, 2016.

[10] P.P. Shen, N.X. Zhou, "Spatio-Temporal evolution and influencing factors of tourism industry efficiency in Jiangsu province:based on DEA-Malmquist index secondary decomposition model," Resources and Environment in the Yangtze Basin, vol. 27, pp. 1, pp. 53-62, 2018.

[11] J. Yuan, Y.H. Liu, "Evaluation of tourism efficiency in the coastal areas of China in the context of the happiness industry," Journal of Coastal Research, vol. 99, pp. 144-150, 2020.

[12] D.C. Wade,M.S. Larry, "Data envelopment analysis (DEA): thirty years on,"European Journal of Operational Research, vol. 192, no. 1, pp. 1-17, 2009.

[13] Y.B. Wang, J.P. Jiang, C.L. Sun, "Study on influential factors of Gansu tourism revenue based on grey relational analysis,” Resource Development \& Market, vol. 31, no. 7, pp.868-871, 2015. 